ARTIGO ORIGINAL

\title{
Compósitos com matriz de gesso reforçada com fibras de madeira de eucalipto
}

\section{Composites with gypsum matrix reinforced with eucalyptus wood fibers}

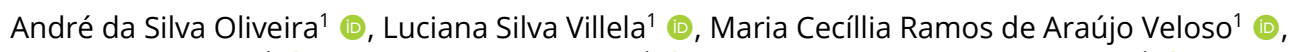

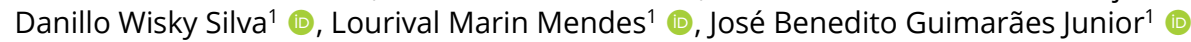 \\ ${ }^{1}$ Universidade Federal de Lavras - UFLA, Lavras, MG, Brasil
}

Como citar: Oliveira, A. S., Villela, L. S., Veloso, M. C. R. A., Silva, D. W., Mendes, L. M., \& Guimarães Junior, J. B. (2020). Compósitos com matriz de gesso reforçada com fibras de madeira de eucalipto. Scientia Forestalis, 48(127), e3260. https://doi.org/10.18671/scifor.v48n127.23

\begin{abstract}
Resumo
O mercado da construção civil assiste a um crescimento na procura por novos materiais, sustentáveis e com melhores propriedades. Este trabalho teve como objetivo desenvolver e caracterizar compósitos à base de gesso reforçados com fibras de eucalipto. Para a produção dos compósitos foram utilizados gesso fino para fundição e fibras de Eucalyptus spp. As proporções utilizadas para a incorporação do reforço foram de $0 ; 2,5 ; 5 ; 7,5$ e $10 \%$ em substituição à massa de gesso. Os corpos de prova foram confeccionados em fôrmas de madeira. Para os ensaios físicos e mecânicos as amostras tiveram dimensões de $40 \times 40 \times 160 \mathrm{~mm}$. As diferentes porcentagens de fibra adicionadas levaram a um aumento em umidade, absorção de água, resistência à flexão estática e resistência à compressão, enquanto na densidade aparente houve decréscimo. Todos os materiais atenderam à normatização para resistência à flexão e compressão. A incorporação das fibras vegetais de eucalipto se mostrou viável para produção de materiais compósitos nos diferentes níveis de reforços avaliados.
\end{abstract}

Palavras-chave: Fibras vegetais; Propriedades físicas; Propriedades mecânicas.

\begin{abstract}
The construction market is growing in the demand for new materials, which are sustainable and present better properties. This work aimed to develop and characterize eucalyptus fiber reinforced plaster based compounds. For the production of composites, thin casting plaster and Eucalyptus spp. fibers were used. The ratios used for the incorporation of reinforcement were $0 ; 2.5 ; 5 ; 7.5$ and $10 \%$ replacing the plaster mass. The specimens were made of wooden molds. Physical and mechanical measurements were taken on samples with dimensions of $40 \times 40 \times 160 \mathrm{~mm}$. With the mixtures strength increased the static bending capacity and compressive strength, while the apparent density decreased. All materials met the standards for bending and compression. The incorporation of eucalyptus plant fibers is a viable alternative for the production of compounds at the different reinforcement levels evaluated.
\end{abstract}

Keywords: Plant fibers; Physical properties; Mechanical properties.

\section{INTRODUÇÃO}

O mercado da construção civil assiste a um crescimento na procura por novos materiais, sustentáveis e com melhores desempenhos, sejam nas propriedades mecânicas, físicas, acústicas ou térmicas (Vila-Chã, 2012). Nesse contexto, destacam-se os materiais compósitos produzidos com fibras vegetais, pois, além de proporcionarem melhoria das propriedades do

Fonte de financiamento: CAPES, FAPEMIG, CNPq.

Conflito de interesse: Nada a declarar.

Autor correspondente: jbguimaraesjr@hotmail.com

Recebido: 24 janeiro 2019.

Aceito: 16 outubro 2019.

Editor: Paulo Henrique Müller Silva.

(c) Este é um artigo publicado em acesso aberto (Open Access) sob a licença Creative Commons Attribution, que permite uso, distribuição e reprodução em qualquer meio, sem restrições desde que o trabalho original seja corretamente citado. 
produto final, são sustentáveis e contribuem para a preservação do meio ambiente (Marinho et al., 2013).

Os produtos à base de gesso utilizados na construção civil vieram ganhando destaque durante as últimas décadas. Porém, embora o gesso tenha propriedades atrativas para a construção civil como ausência de retração por secagem, ótimas propriedades térmicas e acústicas, resistência ao fogo, endurecimento rápido e excelente acabamento superficial, este material ainda apresenta uma série de limitações, como alta permeabilidade à água, natureza porosa, baixa resistência à compressão, baixa resistência à flexão e tração, sendo utilizado somente para ambientes internos (Sophia et al., 2016).

Compósitos são definidos como materiais que apresentam duas ou mais fases distintas. Uma destas fases é contínua (matriz) e a outra é dispersa (Callister Junior \& Rethwisch, 2016; Chawla, 2003; Clyne, 1993).

Ao longo dos últimos anos, muitos estudos, nos quais foi utilizado o gesso como matriz para a produção de compósitos reforçados com partículas e fibras lignocelulósicas, fibras sintéticas e resíduos sólidos, foram publicados. Como exemplo citam-se a incorporação na matriz de gesso fibras de palmeira (Braiek et al., 2017), resíduos de madeira de demolição (Morales-Conde et al., 2016), cinzas de bagaço de cana (Medina Alvarado et al., 2015), abaca (lucolano et al., 2015), fibras de bananeira e papel kraft (Noronha, 2014), fibras têxteis, acrílicas, de vidro, de basalto, de banana e de sisal (Silva et al., 2014), partículas de borracha de pneus reciclados (Serna et al., 2012), granulado de cortiça e fibras têxteis (Camões et al., 2010), fibras lignocelulósicas de bambu, coco e sisal (Magalhães \& Almeida, 2010) e vermiculita e etileno acetato de vinila (eva) (Oliveira, 2009), dentre outros. A utilização desses diferentes reforços não só abrange o conceito de sustentabilidade, como também contribui para o desenvolvimento de novos materiais, com melhores propriedades mecânicas, físicas, térmicas e acústicas.

Fibras vegetais se destacam pelo baixo custo, disponibilidade, baixa densidade, por não serem tóxicas, por apresentar baixa abrasividade aos equipamentos de processo, além de serem renováveis, biodegradáveis e não poluentes (MacVicar et al., 1999; Soykeabkaew et al., 2009). Inúmeras espécies vegetais apresentam potencial para uso como elemento de reforço em compósitos e vêm sendo objetos de estudos, tais como fibras de madeira, bagaço de cana, juta, sisal, bambu, coco e bananeira, entre outras (Mesquita, 2013).

No entanto, falta conhecimento sobre o uso das fibras lignocelulósicas de eucalipto como reforço em compósitos a base de gesso. Estas são produzidas em grande escala no Brasil e no mundo, através de processos mecânicos e termomecânicos, caracterizando um produto com impacto ambiental reduzido e um baixo custo de produção (Silva, 2016).

Com este trabalho, objetivou-se avaliar as propriedades físicas e mecânicas de compósitos à base de gesso com a inserção de fibras de eucalipto spp, visando à produção de materiais com melhores propriedades.

\section{MATERIAL E MÉTODOS}

\section{Caracterização dos materiais utilizados}

Foi utilizado como matriz para produção dos compósitos, gesso fino para fundição proveniente do polo gesseiro do Araripe, no estado de Pernambuco. Esse material apresenta como características massa específica aparente de $781 \mathrm{~kg} / \mathrm{m}^{3}$, tempo de início e fim de pega de 9 e 22 minutos, respectivamente e módulo de finura de 0,85.

O material de reforço utilizado foi as fibras de Eucalyptus spp, provenientes de árvores com 7 anos de idade, coletadas na unidade industrial de produção de Painéis de Fibras de Média Densidade (MDF) da empresa ECTX.SA (Eucatex), Salto de Itu - SP, Brasil.

As fibras foram obtidas pelo processo termomecânico de polpação, onde na passagem dos cavacos pelos rotores é adicionado 0,8\% em massa de emulsão de parafina com intuito de melhorar a estabilidade dimensional das mesmas e diminuir o desgaste dos rotores. 
A relação água/gesso utilizada para a produção dos compósitos foi de 0,6. Tal relação foi escolhida por apresentar boa trabalhabilidade mesmo com o acréscimo das fibras, ficando homogênea, não escorrendo nos moldes e não secando rápido demais. A densidade aparente do gesso foi calculada conforme a NBR 12127 (Associação Brasileira de Normas Técnicas, 1991) e NBR 13207 (Associação Brasileira de Normas Técnicas, 1994).

Os principais atributos anatômicos das fibras foram determinados por microscopia óptica: comprimento médio, largura média e razão de aspecto. A densidade aparente foi determinada levando em consideração a massa da amostra e um volume conhecido. Primeiramente a amostra foi levada à estufa para retirada da umidade. Em seguida, preencheu-se completamente um cadinho de $25 \mathrm{~cm}^{3}$ e foi pesada a massa da amostra que ocupou esse volume. O cálculo foi feito conforme a Equação 1:

$D A=M s / V$

em que:

$\mathrm{DA}=$ Densidade aparente $\left(\mathrm{g} / \mathrm{cm}^{3}\right)$

Ms = Massa seca

$V=$ recipiente com um volume conhecido

Para análise química do material de reforço, as amostras foram armazenadas em frascos e levadas à sala de climatização, em condições de temperatura de $20 \pm 2{ }^{\circ} \mathrm{C}$ e umidade relativa de $65 \pm 3 \%$, até apresentarem massa constante. A análise química foi realizada de acordo com as metodologias e as normas: Cinzas (NBR 13999 - Associação Brasileira de Normas Técnicas, 2003b); Extrativos totais (NBR 14853 - Associação Brasileira de Normas Técnicas, 2010b); Lignina insolúvel (NBR 7989 - Associação Brasileira de Normas Técnicas, 2010a); Holocelulose (por diferença).

\section{Produção dos compósitos minerais}

Foram produzidos compósitos com variação da porcentagem de fibras totalizando cinco diferentes composições (Tabela 1).

Tabela 1. Diferentes composições dos compósitos produzidos.

\begin{tabular}{ccc}
\hline \multirow{2}{*}{ Tratamento } & \multicolumn{3}{c}{ Composição } \\
\cline { 2 - 3 } & Gesso & Fibras \\
\hline 1 & $100 \%$ & $0 \%$ \\
2 & $97,5 \%$ & $2,5 \%$ \\
3 & $95 \%$ & $5 \%$ \\
4 & $92,5 \%$ & $7,5 \%$ \\
5 & $90 \%$ & $10 \%$ \\
\hline
\end{tabular}

Os corpos de prova para os ensaios físicos e mecânicos foram confeccionados com o auxílio de uma forma feita de MDF, fabricada com fundo em madeira de forma a garantir que as dimensões dos corpos de prova fossem de $40 \times 40 \times 160 \mathrm{~mm}$, conforme especificações da EN 13279-2 (European Standards, 2006). Para cada tratamento foram moldados seis corpos de prova a fim de se obter melhor precisão na amostragem.

Após o período de 24 horas os corpos de prova, já completamente sólidos, foram retirados dos moldes e colocados em local bem ventilado e livre da ação de intempéries. Os mesmos foram reorientados diariamente, durante seis dias, para garantir a cura uniforme do gesso. No sétimo dia realizaram-se os ensaios. Na Figura 1 pode-se visualizar os compósitos produzidos 

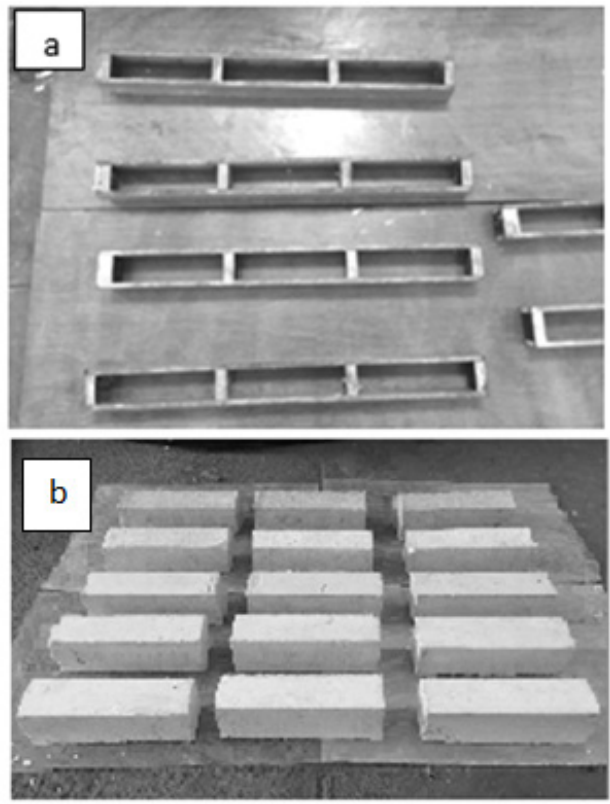

Figura 1. (a) Moldes de confecção dos corpos-de-prova para os ensaios mecânicos e físicos (b) armazenamento dos corpos-de-prova.

\section{Avaliação das propriedades físicas e mecânicas dos compósitos}

Foram adotados os seguintes procedimentos para cada ensaio realizado:

$\checkmark$ Umidade na base seca: NBR 7190 (Associação Brasileira de Normas Técnicas, 1997). $\checkmark$ Densidade aparente: NBR 45 (Associação Brasileira de Normas Técnicas, 2006). $\checkmark$ Ensaio de absorção de água: NBR 14715-2 (Associação Brasileira de Normas Técnicas, 2016) $\checkmark$ Resistência em flexão: EN 13279-2 (European Standards, 2006) $\checkmark$ Resistência em compressão: EN 13279-2 (European Standards, 2006)

\section{Análise dos resultados}

O delineamento utilizado foi o inteiramente casualizado. Para avaliar as variações nas propriedades físicas e mecânicas dos compósitos, estes foram submetidas à análise de variância e regressão, ambas a 5\% de significância.

\section{RESULTADOS E DISCUSSÃO}

A média dos valores encontrados da massa unitária do gesso foi $781,31 \mathrm{~kg} / \mathrm{m}^{3}$, atendendo as especificações da norma NBR 13207 (Associação Brasileira de Normas Técnicas, 1994) que exige massa unitária acima de $700 \mathrm{Kg} / \mathrm{m}^{3}$.

Em relação às propriedades anatômicas das fibras, os valores médios encontrados com seus respectivos desvios estão apresentados na Tabela 2.

Tabela 2. Caracterização anatômica das fibras de eucalipto.

\begin{tabular}{cc}
\hline Caracteristicas anatômicas & Valores médias \\
\hline Comprimento $(\mu \mathrm{m})$ & $979,11 \pm 193,29^{\star}$ \\
Diâmetro $(\mu \mathrm{m})$ & $19,24 \pm 3,12^{\star}$ \\
Parede celular $(\mu \mathrm{m})$ & $4,98 \pm 1,25^{\star}$ \\
Diâmetro do lúmen $(\mu \mathrm{m})$ & $9,11 \pm 3,08^{\star}$ \\
Razão de aspecto & $46,95 \pm 10,69^{\star}$ \\
\hline
\end{tabular}

*Desvio padrão 
Os valores encontrados neste estudo condizem com Gonçalez et al. (2014) que avaliaram a variação de densidade básica da madeira e aspectos anatômicos das fibras de um clone de Eucalyptus urograndis, estes encontraram valor médio de comprimento de fibra de $900 \mu \mathrm{m}$, diâmetro médio da fibra de 16,91 $\mu \mathrm{m}$, largura da parede celular de 5,61 $\mu \mathrm{m}$ e diâmetro do lúmen de $7,12 \mu \mathrm{m}$.

Segundo Savastano Júnior et al. (1997), a relação entre o comprimento e a espessura das fibras influencia a transferência de esforços para a matriz. Quanto maior a razão de aspecto, maior a aderência entre a fibra e a matriz.

Espera-se que os reforços por terem apresentado razão de aspecto alta venham a apresentar maior resistência mecânica, visto que, segundo Iwakiri (2005), a razão de aspecto irá influenciar significativamente a qualidade dos compósitos.

A densidade básica média, com seu respectivo desvio padrão, das fibras de eucalipto foi de $226 \pm 34 \mathrm{~kg} / \mathrm{m}^{3}$, valor este, bem abaixo dos comumente encontrados para madeira de Eucalyptus spp. que oscilam entre 436 e $668 \mathrm{~kg} / \mathrm{m}^{3}$ (Ribeiro \& Zani Filho, 1993). Isso porque na madeira existe um arranjo e a densificação de inúmeras fibras enquanto aqui, trata-se da densidade básica da fibra unitária.

Os resultados das análises químicas das fibras de eucalipto spp estão apresentados na Figura 2.

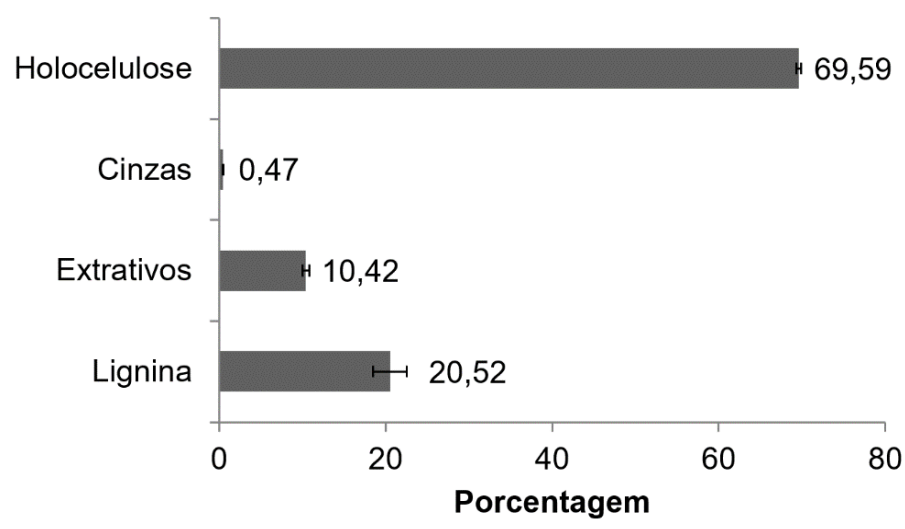

Figura 2. Análises químicas das fibras de eucalipto.

Os valores observados neste trabalho foram próximos ou inferiores aos valores encontrados na literatura. Segundo Sjostrom (1981), Miller (1999), Klock et al. (2005) e Mori et al. (2003) a quantidade de lignina na madeira pode variar entre 23 a 33\%, a quantidade de extrativos pode variar de 5 a 30\%, o teor de cinzas entre 0,2 a $1 \%$ e a quantidade de holocelulose entre 60 a90\%.

Segundo Protásio et al. (2013) os extrativos são um grupo de substâncias químicas heterogêneas relacionadas aos mecanismos de defesa da planta e influenciadas por fatores genéticos e edafoclimáticos. O eucalipto spp apresentou teor de extrativos em média de 6,67\%. Lima et al. (2007) estudaram a madeira de clones de Eucalyptus encontrando valor médio de extrativos totais variando entre 4,79 e 5,54\%. Já Medeiros et al. (2016) encontraram valores médios para o teor de extrativos de $6,51 \%$, sendo este mais próximo com o valor encontrado no presente estudo (10,42\%).

A presença de grande quantidade de extrativos nos materiais lignocelulósicos pode influenciar na interação entre a matriz e o reforço num compósito, uma vez que esses compostos químicos atuam bloqueando os espaços vazios do material lignocelulósico, dificultando o contato com a matriz.

Em relação à lignina, espera-se que seu alto teor em um materialo proporcione maior rigidez, maior resistência à compressão e menor absorção de água visto que, segundo Bhatia et al. (2012), a lignina confere impermeabilidade, rigidez e resistência a ataques microbiológicos e mecânicos aos tecidos vegetais. 
Na Figura 3 pode-se observar os valores médios da umidade na base seca do compósito produzido com inserção de fibras.

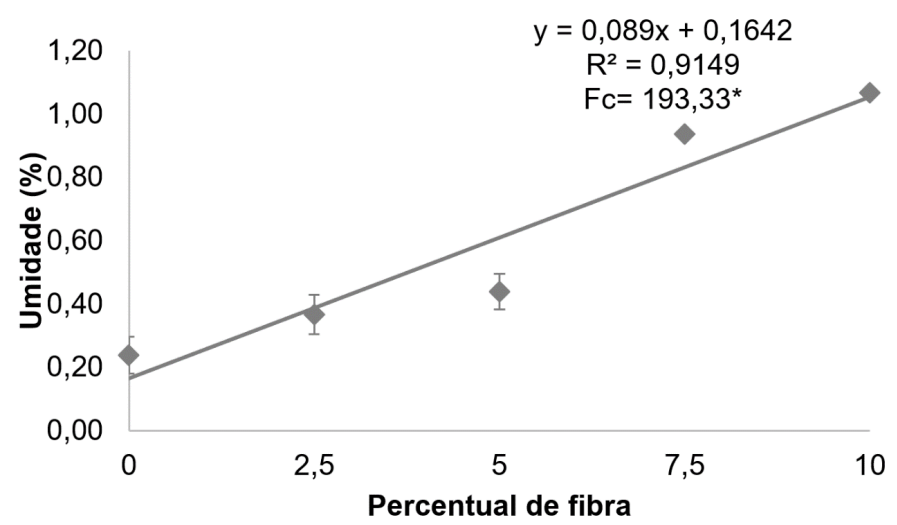

Figura 3. Umidade dos compósitos, onde (*) - significativo ao nível de $5 \%$ de significância.

Os resultados mostram que o aumento da porcentagem de reforços ocasionou um aumento na umidade do compósito, devido a natureza higroscópica das fibras vegetais. Os valores de umidade encontrados variaram de 0,23 a 1,06\%. Observa-se que a umidade não aumentou mais de $2 \%$, o que segundo Chinta et al. (2013) pode ser considerado como controlado.

De acordo com Guimarães Junior et al. (2013) esse fenômeno ocorre porque os materiais lignocelulósicos apresentam grupos hidroxílicos $(\mathrm{OH}-)$ livres, principalmente na região amorfa da celulose e hemiceluloses. Com aumento das porcentagens desses materiais nos compósitos, os mesmos estariam mais propensos a adsorverem umidade.

Chinta et al. (2013) ao estudarem compósitos de gesso reforçados com fibras vegetais (coco, juta, lã, algodão e banana), observaram que à medida que se aumentava a porcentagem de fibras a umidade aumentava gradualmente. Os autores encontraram valores de umidade entre 0,48 a 1,46\%, estando próximos dos encontrados no presente estudo.

Na Figura 4 pode-se observar os valores médios da densidade aparente dos compósitos produzidos com fibras.

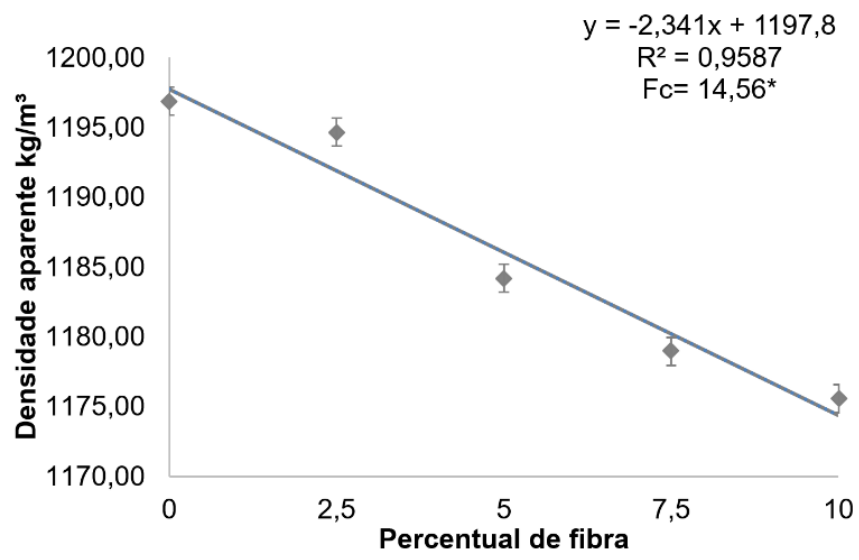

Figura 4. Densidade aparente dos compósitos, onde $\left(^{\star}\right)$ - significativo ao nível de $5 \%$ de significância.

Para a densidade aparente, pode-se observar que, em geral, o aumento da porcentagem de reforços ocasiona redução na densidade da matriz de gesso. A densidade aparente dos compósitos variou de 1175,25 a 1196,96 kg/m³ e o modelo de menor densidade apresentada foi a referência de $10 \%$ de incorporação de Fibra de Eucalipto. Deve se notar que essa 
diminuição ocorre uma vez que a massa especifica do gesso é maior do que o do reforço que foi incorporado.

Rivero et al. (2014) incorporaram borracha na matriz de gesso encontrando para a densidade específica valores entre 880 e $1003 \mathrm{~kg} / \mathrm{m}^{3}$, sendo constatada também diminuição na densidade aparente à medida que se incorporavam partículas de borracha.

Morales-Conde et al. (2016) encontraram valores para a densidade específica entre 602 e $1300 \mathrm{~kg} / \mathrm{m}^{3}$ quando incorporados resíduos de madeira de demolição em compósitos à base de gesso, sendo que, o aumento da porcentagem de aditivos ocasionava uma redução na densidade do gesso.

Khadiran et al. (2016) estudaram a incorporação de diferentes porcentagens de nanocápsulas de n-octadecano em compósitos à base de gesso. Observaram que a densidade aparente diminuía à medida que a porcentagem de massa das nanocápsulas aumentaram devido à menor densidade da nanocápsulas de n-octadecano em comparação com a do gesso. Sendo os valores encontrados de densidade aparente entre 900 a $1900 \mathrm{~kg} / \mathrm{m}^{3}$.

Chinta et al. (2013) estudaram compósitos de gesso reforçados com diferentes fibras naturais (coco, juta, lã, algodão e banana), concluindo que a densidade diminuía quando aumentava o volume das fibras, tornando um material com menos peso. Sendo os valores encontrado de densidade média entre 950 a $1460 \mathrm{~kg} / \mathrm{m}^{3}$.

Na Figura 5 pode-se observar os valores médios da absorção de água dos compósitos produzidos com fibras lignocelulósicas.

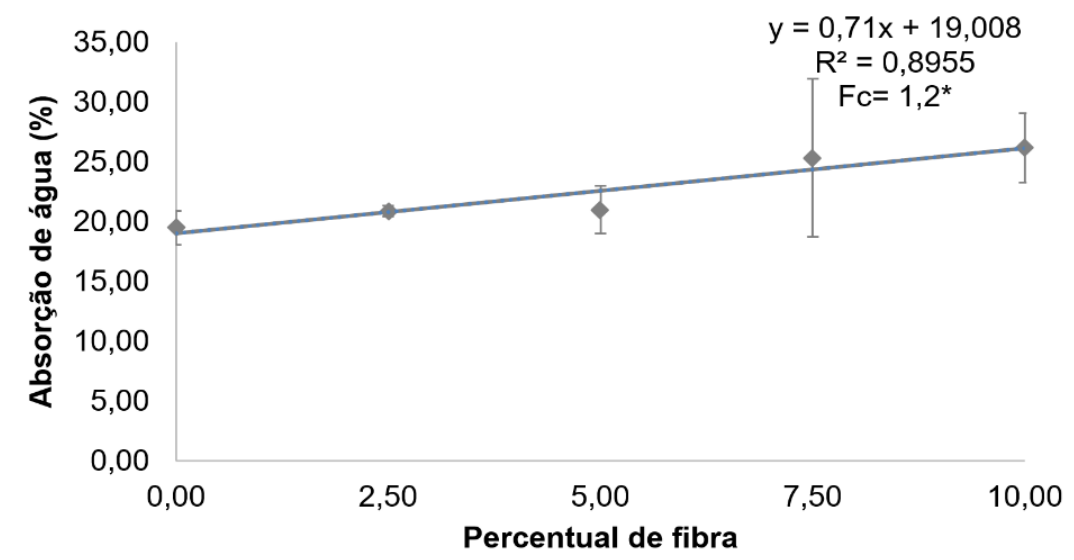

Figura 5. Absorção de água dos compósitos, onde (*) - significativo ao nível de 5\% de significância.

Os resultados mostram que o aumento da porcentagem de reforço ocasionou um aumento na absorção de água, variando de 19,48 a 26,14\%.

Haselein et al. (2002) confeccionaram chapas aglomeradas, utilizando gesso como material cimentante, papel reciclável dissociado (jornal e offset) e partículas de madeira de pinus como reforços. Ao avaliarem a absorção de água, encontraram para a referência valor médio de $28,73 \%$, sendo este valor próximo do encontrado neste estudo para os compósitos com incorporações de reforços $(26,14 \%)$.

Magalhães \& Almeida (2010) pesquisaram um aditivo natural (mucilagem de cactos) adicionado em pastas de gesso. Os autores encontraram valor para a absorção de água do tratamento referência de $27,64 \%$. Eles concluíram que as mucilagens dos cactos estudados diminuíram a taxa de absorção de água dos corpos de prova moldados.

Na Figura 6 pode-se observar os valores médios do módulo de ruptura obtidos para os compósitos em função da adição de diferentes níveis de porcentagens dos reforços. 


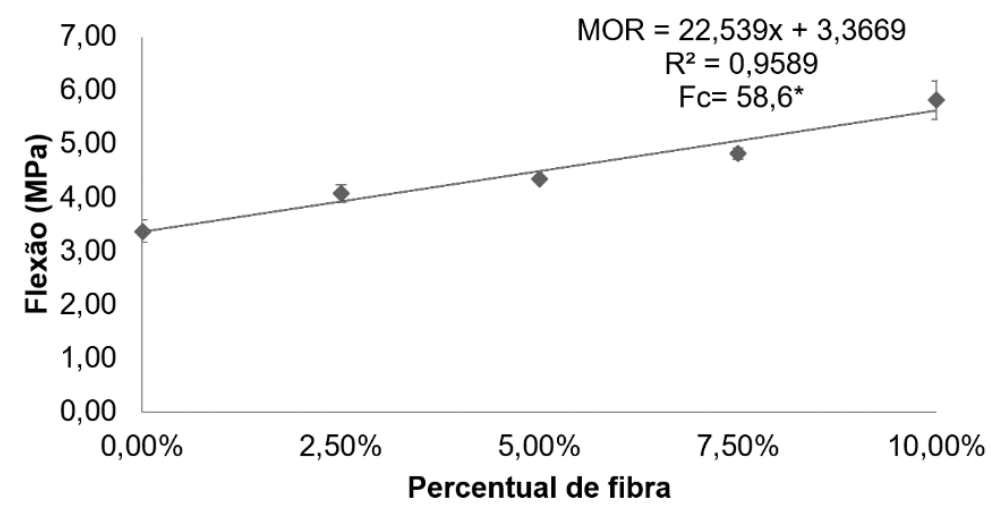

Figura 6. Módulo de ruptura dos compósitos, onde $\left(^{*}\right)$ - significativo ao nível de 5\% de significância.

Os valores encontrados mostram que a resistência à flexão estática aumenta progressivamente com o aumento da porcentagem dos reforços, tendo variação de 3,38 a 5,82 MPa. Observando a média de valores dos compósitos de referência, sem adição de reforços, resultado parecido foi encontrado por Silva et al. (2014) (3,05 Mpa) também para essa condição.

Silva et al. (2014) incorporaram na matriz de gesso 1\% (em massa) de fibras naturais (banana, basalto e sisal) e fibras sintéticas (têxtil, acrílica e de vidro) encontrando valores de resistência à flexão média entre 2,4 a 5,1 MPa, semelhantes ao encontrado neste estudo. Os autores concluíram que as misturas com fibras de vidro e com fibras de basalto apresentaram os melhores resultados de resistência à flexão, eles também relataram que mesmo as fibras dotadas de características mecânicas inferiores conferiram ao compósito um comportamento mais dúctil quando comparados com a mistura sem fibras.

Já lucolano et al. (2015) encontraram valores menores (2,46 a 2,95 Mpa) ao incorporarem diferentes porcentagens de fibras de abacá em matriz de gesso (1, 2 e 3\%).

Todas as porcentagens de incorporação de fibras de eucalipto spp produziram compósitos que cumpriram a norma EN 13279-2 (European Standards, 2006), que define 1,0 MPa como o requisito mínimo para resistência à flexão.

Na Figura 7 pode-se observar os valores médios da resistência máxima à compressão, obtidos para os compósitos em função da adição de diferentes níveis de porcentagens dos reforços.

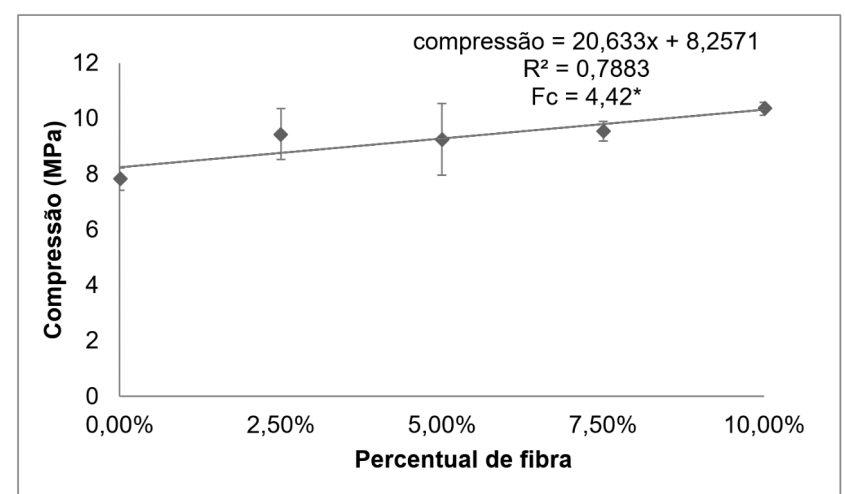

Figura 7. Resistência a compressão dos compósitos, onde (*) - significativo ao nível de 5\% de significância.

Com relação à resistência mecânica à compressão, os valores médios dos compósitos aumentaram à medida em que os reforços foram incorporados. A variação foi de 7,83 a 10,36 MPa, sendo que os compósitos de referência apresentaram o menor valor (7,83 MPa).

Silva et al., (2014) incorporaram na matriz de gesso fibras têxteis e de sisal, observando um comportamento semelhante a este estudo, sendo que, ao adicionarem as fibras, houve aumento na resistência à compressão. 
Ainda, Silva (2010) ao incorporarem 1\% pó de fibra de coco em compósitos de gesso, observaram um aumento de $8,3 \%$ na resistência a compressão.

Todas as porcentagens de incorporação de fibras de eucalipto produziram compósitos que cumpriram a norma EN 13279-2 (European Standards, 2006), que define 2,0 MPa como o requisito mínimo para a resistência à compressão.

\section{CONCLUSÃO}

Pode-se concluir que os reforços adicionados conduziram à diminuição da densidade aparente dos compósitos, resultando em um produto final mais leve. A umidade e absorção de água aumentaram com o aumento das incorporações dos reforços.

Em relação às propriedades mecânicas, os reforços adicionados levaram a um aumento da resistência mecânica, sendo que todos os materiais atenderam normatização para resistência à flexão estática e compressão.

A incorporação das fibras vegetais de eucalipto spp em compósitos à base de gesso se mostrou viável para utilização por exemplo em placas de gesso para divisórias e forros nos diferentes níveis de reforços avaliados, no entanto seriam limitados para ambientes internos e secos, visto que absorveriam mais água.

\section{REFERÊNCIAS}

Associação Brasileira de Normas Técnicas - ABNT. (1991). NBR 12127: gesso para construção: determinação das propriedades físicas do pó: método de ensaio. Rio de Janeiro.

Associação Brasileira de Normas Técnicas - ABNT. (1994). NBR 13207: gesso para construção civil: especificações. Rio de Janeiro.

Associação Brasileira de Normas Técnicas - ABNT. (1997). NBR 7190: projetos de estruturas de madeira. Rio de Janeiro.

Associação Brasileira de Normas Técnicas - ABNT. (2003b). NBR 13999: papel, cartão, pastas celulósicas e madeira: determinação do resíduo (cinza) apos a incineração a $525^{\circ} \mathrm{C}$ (4 p.). Rio de Janeiro.

Associação Brasileira de Normas Técnicas - ABNT. (2006). NBR NM 45: agregados: determinação da massa unitária e do volume de vazios. Rio de Janeiro.

Associação Brasileira de Normas Técnicas - ABNT. (2010a). NBR 7989: pasta celulósica e madeira: determinação de lignina insolúvel em ácido. Rio de Janeiro.

Associação Brasileira de Normas Técnicas - ABNT. (2010b). NBR 14853: madeira: determinação do material solúvel em etanol-tolueno e em diclorometano e em acetona (3 p.). Rio de Janeiro.

Associação Brasileira de Normas Técnicas - ABNT. (2016). Dossiê Técnico Drywall - Chapas de Gesso, Convênio Sebrae ABNT. NBR 14715-2:2010: chapas de gesso para drywall: parte 2: métodos de ensaio. São Paulo.

Bhatia, L., Johri, S., \& Ahmad, R. (2012). An economic and ecological perspective of ethanol production from renewable agro waste: a review. AMB Express, 2(1), 65. PMid:23217124. http://dx.doi.org/10.1186/2191-0855-2-65.

Braiek, A., Karkri, M., Adili, A., Ibos, L., \& Ben Nasrallah, S. (2017). Estimation of the thermophysical properties of date palm fibers/gypsum composite for use as insulating materials in building. Energy and Building, 140, 268-279. http://dx.doi.org/10.1016/j.enbuild.2017.02.001.

Callister Junior, W. D., \& Rethwisch, D. G. (2016). Ciência e engenharia de materiais: uma introdução (9. ed.). Rio de Janeiro: LTC.

Camões, A., Cardoso, C., Cunha, S., Vasconcelos, G., Medeiros, P., Eires, R., Jalali, S., \& Lourenço, P. (2010). Caracterização de materiais compósitos à base de gesso FGD. In Anais do $8^{\circ}$ Congresso Nacional de Mecânica Experimental (1 CD-ROM). Minho: Universidade do Minho.

Chawla, K. K. (2003). Ceramic matrix composites (2nd ed.). Birmingham: KAP. http://dx.doi.org/10.1007/978-1-4615-1029-1.

Chinta, S. K., Katkar, P. M., \& Jafer, M. M. (2013). Natural fibres reinforced gypsum composites. International Journal of Engineering and Management Sciences, 4(3), 318-325.

Clyne, T. A. (1993). An introduction to metal matrix composites. Cambridge: Cambridge University Press. . http://dx.doi.org/10.1017/CBO9780511623080. 
European Standards. (2006). EN 13279-2: gypsum binders and gypsum plasters, part 2: test methods. Pilsen.

Gonçalez, J. C., Santos, G. L., Silva Júnior, F. G., Martins, I. S. M., \& Costa, J. A. (2014). Relações entre dimensões de fibras e de densidade da madeira ao longo do tronco de Eucalyptus urograndis. Scientia Forestalis, 42(101), 81-89.

Guimarães Junior, J. B., Mendes, L. M., Mendes, R. F., Guimarães, B. M. R., \& Melo, R. R. (2013). Efeito do teor de parafina nas propriedades físico-mecânicas de painéis aglomerados de Pinus oocarpa. Ciência da Madeira, 4(1), 72-82. http://dx.doi.org/10.12953/2177-6830.v04n01a06.

Haselein, C. R., Calegari, L., Alberti, L. F., Minello, A. L., Silva, P. A., \& Pintos, R. G. F. (2002). Fabricação de chapas de partículas aglomeradas usando gesso como material cimentante. Ciência Florestal, 12(1), 81-88. http://dx.doi.org/10.5902/198050981703.

lucolano, F., Caputo, D., Leboffe, F., \& Liguori, B. (2015). Mechanical behavior of plaster reinforced with abaca fibers. Construction \& Building Materials, 99, 184-191. http://dx.doi.org/10.1016/j.conbuildmat.2015.09.020.

Iwakiri, S. (2005). Painéis de madeira reconstituída (247 p.). Curitiba: FUPEF.

Khadiran, T., Hussein, M. Z., Syamsi, H. W., Zainal, Z., \& Rusli, R. (2016). Laboratory-scale studies on smart gypsum composite boards incorporated with nano-encapsulated organic phase change material for thermal comfort building application. Journal of Materials in Civil Engineering, 28(3), 04015137. http://dx.doi.org/10.1061/(ASCE)MT.1943-5533.0001394.

Klock, U., Muñiz, G. I. B., Hernandez, J. A., \& Andrade, A. S. (2005). Química da madeira (3. ed.). Curitiba: UFPR.

Lima, C. K. P., Mori, F. A., Mendes, L. M., \& Carneiro, A. C. O. (2007). Características anatômicas da madeira de clones de Eucalyptus e sua influência na colagem. Cerne, 13(2), 123-129.

MacVicar, R., Matuana, L. M., \& Balatinecz, J. J. (1999). Agingmechanism in celulose fiber reinforced cement composites. Cement and Concrete Composites, 21(3), 189-196. http://dx.doi.org/10.1016/S0958-9465(98)00050-X.

Magalhães, A. C. T. V., \& Almeida, J. G. (2010). O uso da mucilagem de cacto em pastas de gesso: efeitos na absorção de água e na resistência à flexão estática. Ambiente Construído, 10(1), 139-151. http://dx.doi.org/10.1590/S1678-86212010000100008.

Marinho, N. P., Nascimento, E. M., Nisgoski, S., Magalhães, W. L. E., Claro Neto, S., \& Azevedo, E. C. (2013). Caracterização física e térmica de compósito de poliuretano derivado de óleo de mamona associado com partículas de bambu. Polímeros, 23(2), 201-205.

Medeiros, B. L. M. A., Guimarães Junior, J. B., Ribeiro, M. X., Lisboa, F. J. N., Guimarães, I. L., \& Protásio, T. P. (2016). Avaliação das propriedades físicas e químicas da madeira de Corymbia citriodora e Eucalyptus urophylla x Eucalyptus grandis cultivadas no Piauí. Nativa, 4(6), 403-407. http://dx.doi.org/10.14583/2318-7670.v04n06a10.

Medina Alvarado, R., Burneo Valdivieso, X., Hernández-Olivares, F., \& Zuñiga Suárez, A. (2015). Reuse of organic waste type in the development of ecoefficient and sustainable composites. In Annali del 2 Congreso Internacional de Construcción Sostenible y Soluciones Ecoeficientes (1 CD-ROM). Sevilla: Universidad de Sevilla.

Mesquita, R. G. A. (2013). Inclusão de materiais lignocelulósicos na produção de compósitos plásticos (Dissertação de mestrado). Universidade Federal de Lavras, Lavras.

Miller, R. B. (1999). Wood handbook: wood as an engineering material (463 p.). Madison: Forest Products Laboratory.

Morales-Conde, M. J., Rodríguez-Liñán, C., \& Pedreño-Rojas, M. A. (2016). Physical and mechanical properties of wood-gypsum composites from demolition material in rehabilitation works. Construction \& Building Materials, 114, 6-14. http://dx.doi.org/10.1016/j.conbuildmat.2016.03.137.

Mori, F. A., Mendes, L. M., Trugilho, P. F., \& Cardoso, M. G. (2003). Utilização de eucaliptos e de madeiras nativas no armazenamento da aguardente de cana-de-açúcar. Food Science and Technology, 23(3), 396-400. http://dx.doi.org/10.1590/S0101-20612003000300018.

Noronha, L. L. (2014). Elementos construtivos e de proteção térmica à base de gesso e resíduos agroindustriais (Dissertação de mestrado). Universidade Federal da Paraíba, João Pessoa .

Oliveira, M. (2009). Materiais compósitos à base de gesso contendo EVA (etileno acetato de vinila) e vermiculita: otimização e propriedades termodinâmicas (Tese de doutorado). Universidade Federal de Paraíba, João Pessoa. 
Protásio, T. P., Bufalino, L., Guimarães Junior, M., Tonoli, G. H. D., \& Trugilho, P. F. (2013). Técnicas multivariadas aplicadas a avaliação de resíduos lignocelulosicos para a produção de bioenergia. Ciência Florestal, 23(4), 771-781. http://dx.doi.org/10.5902/1980509812361.

Ribeiro, F. A., \& Zani Filho, J. (1993). Variação da densidade básica da madeira em espécies/procedências de Eucalyptus spp (No. 46, pp. 76-85). Piracicaba: IPEF.

Rivero, A. J., Guzmán Báez, A., \& Navarro, J. G. (2014). New compositegypsumplaster-groundwaste rubber comingfrompipefoaminsulation. Construction \& Building Materials, 55, 146-152.

Savastano Júnior, H., Agopyan, V., \& Oliveira, L. (1997). Estudo da microestrutura das fibras vegetais e de suas implicações sobre o desempenho de compósitos cimentícios. Revista Engenheiro Agrícola, 17(1), 113-124.

Serna, A., Río, M., Palomo, J. G., \& González, M. (2012). Improvement of gypsum plaster strain capacity by the addition of rubber particles from recycled tyres. Construction \& Building Materials, 35, 633641. http://dx.doi.org/10.1016/j.conbuildmat.2012.04.093.

Silva, D. W. (2016). Propriedades físico-mecânicas de fibrocimento reforçado com fibras de eucalipto tratadas termicamente (Dissertação de mestrado). Universidade Federal de Lavras, Lavras.

Silva, L., Camões, A., \& Vasconcelos, G. (2014). Material compósito à base de gesso reforçado com fibras: caracterização mecânica. Azurém: Departamento de Engenharia Civil, Universidade do Minho.

Silva, M. G. S. (2010). Desenvolvimento de compósitos a base de gesso e pó de fibras de coco (Dissertação de mestrado). Universidade Federal de Sergipe, São Cristóvão. Recuperado em 23 de dezembro de 2018, de http://www.ebookcult.com.br/?acao=ver.livro\&id=837 46

Sjostrom, S. (1981). Wood chemistry: fundamentals and applications. London: Academic.

Sophia, M., Sakthieswaran, N., \& Babu, G. O. (2016). Gypsum as a construction material: a review of recent developments. International Journal for Innovative Research in Science \& Technology, 2(12), 1-9.

Soykeabkaew, N., Sian, C., Gea, S., Nishino, T., \& Peijs, T. (2009). All-cellulose nanocomposites by surface selective dissolution of bacterial cellulose. Cellulose, 16(3), 435-444. http://dx.doi.org/10.1007/s10570-009-9285-1.

Vila-Chã, N. (2012). Estudo de um material compósito à base de gesso e cortiça (Dissertação de mestrado). Universidade do Minho, Braga.

Contribuição dos Autores: ASO: Curadoria de Dados, investigação, metodologia, escrita- primeira redação, Administração do Projeto; LSV: Escrita- Revisão e Edição, Supervisão; MCRAV: Visualização, Supervisão; DWS: Supervisão; LMM: Supervisão, Validação, Obtenção do Financiamento; JBGJ:Supervisão, Validação, Obtenção do Financiamento, Análise formal. 